\title{
Cost-effective traffic scheduling for Cloud resource management
}

\begin{abstract}
Cloud resource management experienced massive strategy development throughout recent years. A traffic scheduling pattern becomes more significant in Cloud for adapting heterogeneous processing demands. We present priority-based traffic scheduling for downlink resource management aims to sustain Quality of Services (QoS) in Cloud which provide Cost-Effective Best Effort service (CEBE). Our traffic scheduling embraces two main functions are priority assignment and resource bandwidth allocation. Specifically, we leverage prioritization for several downlink services, and then dynamically allocate suitable bandwidth for improving accessibility while providing efficient communication cost. Our simulation results illustrate that priority-based traffic scheduling has more substantial performance in terms of throughput and delay than others.
\end{abstract}

Keyword: Bandwidth allocation; Cloud computing; Priorities; QoS; Resource management; Traffic scheduling 